New Drugs

\title{
New approaches in managing drug overdosage and poisoning
}

\author{
L F PRESCOTT
}

The incidence of drug overdosage and poisoning has continued to increase over the past decade, and this form of self induced disease imposes a major burden on the health services. Unfortunately, specific antidotes are available for very few drugs or poisons, and treatment (at least for the deeply unconscious patient) depends largely on supportive treatment based on the standard principles of intensive care. In this context there have been important advances with the spread of intensive care units and the general availability of facilities for comprehensive monitoring and cardiorespiratory support. New developments in drug treatment include the use of agents such as dopamine for maintaining renal function in the presence of hypotension.

With a better understanding of the mechanisms of drug action and the application of pharmacokinetic principles there have been other important advances in the management of poisoning, particularly in the use of specific antidotal treatment and specialised techniques for drug removal such as haemoperfusion. In addition, improvements in drug analytical methodology have allowed a more critical evaluation of established procedures for the enhancement of drug elimination.

In one sense some advances in management have been negative. Thus physostigmine is no longer recommended for anticholinergic poisoning, and the general use of techniques such as forced diuresis and dialysis has declined greatly. The advantages of a conservative approach with a minimum of meddlesome medical interference are becoming more widely appreciated.

\section{Trends in the patterns of self poisoning}

There have been important changes over the years in the drugs and poisons taken. Self poisoners usually take on impulse what happens to be available at the time of crisis, and what is available is often what has recently been prescribed in attempts to alleviate distress caused by underlying personal problems. Not surprisingly, the drugs available change with the fashions of prescribing. Over the past 15 years there has been a welcome decline in the use of the highly dangerous barbiturate hypnotics which have been replaced by the safer but ubiquitous benzodiazepines. The need for the full resources of intensive care in the management of poisoning has been correspondingly reduced, but new problems have emerged, particularly with Distalgesic (paracetamol plus the narcotic analgesic d-propoxy-

Regional Poisoning Treatment Centre and University Department of Therapeutics and Clinical Pharmacology, Royal Infirmary, Edinburgh EH3 9YW

L F PRESCOTT, MD, FRCPED, consultant physician phene), tricyclic antidepressants, chlormethiazole, beta-blocking drugs, quinine, aminophylline, lithium salts, and the infamous weedkiller, paraquat. The incidence of paracetamol poisoning has increased greatly over the past 15 years, but effective treatment is now available. The widely prescribed combination of paracetamol with d-propoxyphene (Distalgesic) is espcially dangerous, however, and a relatively small overdose can cause fatal respiratory depression with alarming rapidity, particularly if taken with ethanol. In Birmingham and the West Midlands (and probably other areas also) Distalgesic has become the foremost cause of death by drug overdosage.

Young children and toddlers have a habit of putting anything they find into their mouths, including brightly coloured medicines and various household products. The amounts taken, however, are usually small, and serious problems are relatively uncommon. Regrettably, older children are adopting the adult patterns of self poisoning and drug abuse on an increasing scale.

\section{Reduction in gastrointestinal absorption}

Gastric aspiration and lavage with a wide-bore tube is the preferred method for removing unabsorbed drug or poison, but it is hazardous if the protective pharyngeal reflexes are obtunded and if corrosives or liquid hydrocarbons have been taken. It is also psychologically traumatic in young children. There is an increasing tendency to use emetics as an alternative, and syrup of ipecac is the current favourite. Emetics such as saline and copper sulphate solution can be lethal and should never be used. Controversy continues concerning the relative efficacy of gastric lavage and induced emesis, and all that may be said is that neither can be relied on to empty the stomach completely.

The administration of activated charcoal is increasingly advocated in the belief that absorption will be limited by the binding of unabsorbed drug in the gut. Although charcoal is a powerful adsorbent, it has little or no effect unless given in large amounts within an hour of ingestion of the drug or poison. Unfortunately, most patients arrive at hospital far too late for it to act in this way, and we do not use it.

On the other hand, charcoal and cholestyramine given by mouth in repeated doses may interrupt the enterohepatic circulation of polar high molecular weight compounds that are excreted into bile, thus preventing their intestinal reabsorption. By this means the elimination of drugs such as phenobarbitone, digitoxin, and phenprocoumon can be considerably enhanced after overdosage. Similar effects have been reported with charcoal in healthy volunteers given therapeutic doses of drugs such as phenylbutazone, but its efficacy in severely poisoned patients is likely to be limited by slow gastric emptying, reduced gastrointestinal motility, or vomiting. Nevertheless, such treatment is cheap, simple, and safe, and properly controlled studies in poisoned patients are required. 


\section{Enhancement of drug removal from the body}

The most important change in the enhancement of drug removal from the body has been a long overdue decline in the uncritical and indiscriminate use of forced diuresis and dialysis. These measures have a limited application in the management of poisoning, and a better understanding of the relevant physiological and pharmacokinetic principles has led to a more selective and realistic approach to their use. Forced alkaline diuresis is now largely restricted to moderate to severe poisoning with salicylates and phenobarbitone. It is also effective in removing the selective weedkiller 2,4-dichlorophenoxyacetic acid $(2,4-\mathrm{D})$. There is a dearth of information concerning the efficacy of forced acid diuresis in poisoned patients and it is rarely used. Contrary to established teaching it does not usefully enhance the elimination of quinine after overdosage.

There are particular problems with the use of forced alkaline diuresis for salicylate poisoning. Like other anti-inflammatory drugs that inhibit prostaglandin synthesis, salicylate in overdosage causes retention of sodium and water and in some patients renal function is impaired. The resultant retention of fluid is potentially hazardous, and haemodilution causes a spurious fall in plasma salicylate concentrations, which gives a misleading impression of efficacy. The renal clearance of salicylate depends much more on urine $\mathrm{pH}$ than flow rate, and alkalinisation of the urine is therefore more important than attempts to force a diuresis.

Haemodialysis is occasionally indicated in severely poisoned patients for removal of compounds of low molecular weight that are not extensively bound to plasma proteins and have a small volume of distribution and a low spontaneous clearance. Many drugs and poisons may now be removed more effectively by charcoal or ion exchange resin haemoperfusion, but haemodialysis may be preferred if there are acid base, fluid, or electrolyte disturbances as, for example, in salicylate poisoning. Haemodialysis is useless for removing paraquat. The clearance of drugs by peritoneal dialysis is low and it has virtually been abandoned in the management of poisoning. It does, however, have an advantage over haemodialysis in that it may be used continuously over several days if necessary.

Haemoperfusion is a recently introduced technique in which heparinised blood from the patient is passed through a column containing coated charcoal or exchange resin and then returned to the patient. It is technically easier to perform than haemodialysis, and the initial clearances of drugs such as barbiturates, carbamazepine, glutethimide, meprobamate, salicylate, theophylline, and paracetamol are as good or better than those achieved by haemodialysis. The removal of drugs and poisons by exchange resin is probably more selective than with charcoal, but both may remove endogenous compounds and both may cause appreciable thrombocytopenia. Drug clearance usually falls progressively during haemoperfusion, and the duration of treatment is normally limited to six to eight hours. The same basic limitations for drug removal from the body apply to haemoperfusion as to haemodialysis, and not all drugs and poisons can be removed. Thus charcoal haemoperfusion is not effective in removing quinine, quinidine, camphor, or diphenylhydantoin. Most poisoned patients recover with intensive supportive treatment alone, and the indications for haemoperfusion are few and far between. It may occasionally be indicated in very severe poisoning and in patients with serious complications such as pneumonia or renal failure.

\section{Naloxone for narcotic overdosage}

The management of intoxication with narcotic analgesics has been transformed by the introduction of the specific antagonist naloxone. Unlike its predecessors nalorphine and levallorphan, naloxone has no partial agonist activity and may be used without fear of aggravating respiratory depression. Naloxone is effective in reversing the depressant effects of not only morphine, heroin, and pethidine but also other opiates such as d-propoxyphene (present in Distalgesic), dihydrocodeine (DF118), methadone, dipipanone (present in Diconal), dextromoramide, and diphenoxylate (present in Lomotil).

Naloxone is a competitive antagonist, and much larger doses are required for the full reversal of severe narcotic overdosage than those recommended by the manufacturer. The first adult dose should be not less than 1.2-1.6 mg intravenously and since it has a very short duration of action, repeated large doses may have to be given. Even larger doses may be required for severe poisoning with partial agonists which have a high affinity for receptors such as pentazocine. The recent introduction of the partial agonist buprenorphine is particularly worrying since its depressant effects cannot be readily reversed with naloxone. Naloxone has been reported to produce minor but variable arousal in some patients with benzodiazepine or ethanol intoxication, but there are no indications for its use in such circumstances. It may produce a transient acute withdrawal reaction in narcotic addicts.

\section{$\mathrm{N}$-acetylcysteine and methionine for paracetamol poisoning}

One of the most important developments in the management of poisoning has been the recent introduction of sulphydryl compounds such as $\mathrm{N}$-acetylcysteine and methionine for the treatment of paracetamol poisoning. The major complication of paracetamol poisoning is acute hepatic necrosis, and this is caused by the formation of a highly reactive intermediate metabolite that is normally removed by conjugation with hepatic reduced glutathione. Liver damage after paracetamol overdosage occurs only when glutathione becomes depleted, and these protective agents probably act mainly by facilitating glutathione synthesis. Paracetamol poisoning was first treated successfully with cysteamine in 1973, but this agent has since been abandoned because of its toxicity and intravenous $\mathrm{N}$ acetylcysteine is currently the treatment of choice.

Paracetamol is rapidly metabolised, and treatment must be started within 8-10 hours of ingestion to be effective. The protective effect falls off increasingly rapidly after this time, and treatment after 15 hours is not only ineffective but potentially dangerous. Most unselected patients referred to hospital after overdosage of paracetamol are not at risk of appreciable liver damage. Without specific treatment, only about $8 \%$ develop severe liver damage with a raised plasma asparate or alanine aminotransferase activity above $1000 \mathrm{IU} / \mathrm{l}$, about $1 \%$ develop acute renal failure, and $1-2 \%$ die in hepatic failure. It is therefore essential to identify the few patients who require treatment, and this can be done only by emergency estimation of the plasma paracetamol concentration, since apart from nausea and vomiting there are no specific early clinical symptoms of severe intoxication. Treatment is indicated in patients with plasma paracetamol concentrations above a line on a semilogarithmic graph joining plots of $200 \mu \mathrm{g} / \mathrm{ml}$ at four hours and $30 \mu \mathrm{g} / \mathrm{ml}$ at 15 hours. $\mathrm{N}$-acetylcysteine appears to be more effective than methionine, and intravenous administration is essential because most severely poisoned patients develop nausea and vomiting within a few hours of ingestion of the paracetamol. $\mathrm{N}$-acetylcysteine may rarely cause a minor transient allergic reaction, and methionine given late may precipitate or aggravate hepatic failure.

No form of late treatment (after 15 hours) has been shown to reduce the severity of liver damage or prevent hepatic failure. Recent claims for the efficacy of late charcoal haemoperfusion for paracetamol poisoning must be viewed with caution. In one report haemoperfusion was started in seven patients on average 23 hours after the drug was taken, and the mean amount removed was $2.4 \mathrm{~g}$. One patient died in hepatic failure and as judged by the plasma bilirubin concentration and prothrombin time ratio five developed severe liver damage. The amounts of paracetamol removed in the other two patients were only 0.38 and $0.36 \mathrm{~g}$ (equivalent to less than one tablet). 


\section{Physostigmine for anticholinergic poisoning}

Physostigmine is a short acting reversible cholinesterase inhibitor that has been used with enthusiasm in some quarters for the reversal of intoxication with anticholinergics, notably the tricyclic antidepressants. It is given in the form of physostigmine salicylate, usually intravenously. While it can undoubtedly reverse coma and delirium induced by these drugs its effects are short lived, and the dose must be carefully titrated. Unfortunately, it may cause convulsions, and excess cholinergic activity can cause serious complications including asystole. Furthermore, there is no good evidence that physostigmine reverses the serious cardiac arrhythmias with conduction defects and the myocardial depression associated with severe tricyclic antidepressant poisoning. The use of physostigmine cannot therefore be recommended.

\section{Glucagon for poisoning with beta-blocking drugs}

Severe intoxication with beta-blockers such as propranolol and oxprenolol may produce bradycardia, hypotension, and gross myocardial depression with cardiogenic shock. In the latter circumstances the officially recommended treatment with atropine and isoprenaline is virtually useless. Atropine has no effect, and very large doses of the specific antagonist isoprenaline have to be given. The dose has to be carefully titrated, and in the meantime the patient may die. The use of glucagon may avoid these problems. Glucagon is a cardiac stimulant, its action is not inhibited by beta-blockade, and unlike isoprenaline in excessive dosage it does not cause cardiac arrhythmias. Initial intravenous bolus injections of $5-10 \mathrm{mg}$ of glucagon may be followed by infusion at a rate sufficient to maintain an adequate cardiac output. Hyperglycaemia does not seem to be a problem, but vomiting may occur.

\section{Immunotherapy}

The administration of specific antibodies to bind and inactivate a drug or poison at the sites of action is an interesting new development in the treatment of poisoning that is still in its infancy. It is expensive and probably only practicable with agents such as digoxin and paraquat, which produce toxicity at low tissue concentrations. Severe digoxin poisoning has been treated successfully with Fab fragments of sheep digoxin-specific antibodies, but such treatment is not generally available in the United Kingdom. It is interesting to speculate on the possibility that patients who poison themselves repeatedly could be "immunised" against the toxic effects of their favourite drugs and poisons.

\section{Bibliography}

Proudfoot AT, Park J. Changing pattern of drugs used for selt-poisoning. Br Med F 1978;i:90-3.

A description of the changing morbidity and pattern of drugs used for self poisoning over the years in Edinburgh.

Whittington RM, Barclay AD. The epidemiology of dextropropoxyphene (Distalgesic) overdose fatalities in Birmingham and the West Midlands. $\mathcal{F}$ Clin Hosp Pharm $1981 ; 6: 251-7$.

Yet another strong reminder of the lethal properties of Distalgesic taken in overdosage.

Prescott LF. Drug overdosage and poisoning. In: Avery GS, ed. Drug treatment. 2nd ed. Sydney: Adis Press, 1980:263-81.

A review of the pharmacological principles concerned in the treatment of poisoning, including specific treatment and limitations of forced diuresis, dialysis, and haemoperfusion.

Proudfoot AT. Diagnosis and management of acute poisoning. Edinburgh: Blackwell Scientific Publications, 1982.

An authoritative, up to date, and concise guide to the management of poisoning based on extensive personal clinical experience.

Vale JA, Meredith TJ, eds. Poisoning diagnosis and treatment. London: Update Books, 1981

A topical account of the diagnosis and treatment of clinically important poisoning with an emphasis on mechanisms of toxicity.

Prescott LF. Paracetamol overdosage: pharmacological considerations and clinical management. Drugs 1983;25:290-314.

A detailed review of the mechanisms of paracetamol hepatotoxicity and clinical management of overdosage including the use of specific antidotal treatment.

Smith TW, Haber E, Yeatman L, Butler VP. Reversal of advanced digoxin intoxication with $\mathrm{Fab}$ fragments of digoxin-specific antibodies. $N$ Engl f Med 1976;294:797-800.

A detailed description of dramatic amelioration of severe digoxin intoxication after the administration of digoxin-specific antibodies.
Is the evidence for using anticoagulants in the treatment of deep vein thrombosis anecdotal or is it based on controlled clinical trials?

Not much of the evidence for the use of anticoagulants in venous thrombosis is based on controlled trials. Heparin was first introduced into therapeutic use in Sweden and Canada in the late 1930s, before the days of randomised double blind controlled trials. Nevertheless, several careful clinical studies offered such strong evidence for the therapeutic value of heparin that there have been ethical objections to any subsequent trial that would have included an untreated control group. ${ }^{12}$ One such trial was reported in 35 patients with pulmonary embolism in 1960 by Barritt and Jordan. ${ }^{3}$ It was discontinued after five deaths and five recurrences had occurred in the no treatment group and no deaths or recurrences, or both, in the heparin treated group. There is no doubt that heparin is a highly effective anticoagulant and that it is the treatment of choice in acute venous thrombosis and embolism-unless thrombolytic treatment is preferred. Heparin should be continued for seven to 10 days and should be overlapped with oral anticoagulants for four to five days, not just the 48 hours which is so commonly practised. The use of oral anticoagulants is also based largely on uncontrolled studies. This is hardly surprising when you think of the practical difficulties in conducting such a trial. Kakkar et al, however, compared eight patients given oral anticoagulants for six to nine months with six patients who received none. ${ }^{4}$ The former group showed better recanalisation on follow up phlebography. Pathological studies have shown that recanalisation may take very many months and that patients are likely to have recurrent episodes. It therefore seems logical to try to reduce the likelihood of recurrent thrombosis by treating with anticoagulants during this period, especially as one of the objectives should be to prevent the development of a post-thrombotic syndrome. My practice is to treat with oral anticoagulants and knee length stockings for at least six months and to continue longer if post-thrombotic swelling is still present.-C V RUCKLEY, consultant vascular surgeon, Edinburgh.

' Zilliacus II. On specific treatment of thrombosis and pulmonary embolism with anticoagulants, with particular reference to post-thrombotic sequelae: results of 5 years treatment of thrombosis and pulmonary embolism at a series of ${ }^{2}$ Bauer G. Thrombosis: early diagnosis and abortive treatment with heparin. Lancet $1946 ; \mathrm{i}: 447-54$.

${ }^{3}$ Barritt DW, Jordan SC. Anticoagulant drugs in the treatment of pulmonary embolism. Lancet 1960;i:1309-12.

Kakkar VV, Howe CT, Laws JW, Flane C. Late results of treatment of deep vein thrombosis. Br Med $\mathcal{F} 1969 ; \mathrm{i}: 810-1$.

\section{What health hazards are associated with swimming in the Thames?}

Apart from the common hazards of drowning and trauma infection is the greatest danger. In the non-tidal reaches of the river the risks are slight, as the Thames Water Authority makes strenuous efforts to control the sewage effluents from upstream townships and any pathogens present will be well diluted. Trade and radioactive discharges are also carefully monitored. There is little danger from salmonella infections. Viruses may be present in infective doses, however, and swimmers should have the protection of poliomyelitis vaccine. I know of no cases of virus hepatitis from swimming in non-tidal waters, and Weil's disease is not likely to occur. Eye and ear infections are not a real hazard. In the tidal reaches adjoining canals and docks the water and river banks may be heavily polluted, and intestinal infections, viral diseases, together with eye and ear infections, could be a real hazard.-ANDREW B SEMPLE, emeritus professor of community and environmental health, Liverpool. 